\title{
Preparation of vinyl chloride - vinyl ether copolymers via partial etherification from PVC
}

\author{
H. Mekki*, M. Belbachir \\ Laboratoire de Chimie des Polymères, Département de Chimie, Faculté des Sciences, Université d'Oran Es-Senia, BP N \\ 1524, El M’ Naouer, Oran 31000, Algérie
}

Received 2 April 2007; accepted in revised form 21 June 2007

\begin{abstract}
The chemical modifications of poly (vinyl chloride) with aliphatic and aromatic alcohols compounds have been investigated at room temperature and atmospheric pressure, catalysed by a new green basic catalyst, the Maghnite- $\mathrm{K}^{+}$. The presence of ether groups in the products is proven by infra red spectroscopy (IR) as well as by nuclear magnetic resonance spectroscopy ( $\left.{ }^{1} \mathrm{H} \mathrm{NMR}\right)$, and characterized by intrinsic viscosity as well as by gel permeation chromatography (GPC).
\end{abstract}

Keywords: polymer membranes, Maghnite-K, PVC, grafting copolymers, Algerian clay, basic catalyst

\section{Introduction}

The purpose of this work was to highlight the chemical interaction of poly (vinyl chloride) with diverse alcohols and trying to introduce them onto polymer chain.

PVC is one of the most important commercial polymers due to its low production costs and its excellent stability to acids and bases [1]. However, there is some shortcoming such as limited thermal and mechanical stability or sensitivity to ultraviolet radiation [2].

By chemical modifications reactions of PVC with appropriate compounds, new polymers with improved physical properties can be obtained $[3,4]$.

The nucleophilic substitution of chlorine atoms with thiophenolate [1], amine [5] and thiol [6] has been intensively studied in recent years.

On the other hand, it has recently been shown $[6,7]$ that PVC can also be used as an interesting starting membrane material for gas separation when the polymer is chemically modified.
In the present communication, we show that reaction of PVC with alcohols catalysed by Maghnite-K $\mathrm{K}^{+}$ in THF solution gives a new white copolymer (VCEther) product which is soluble in DMF, DMSO, partially soluble in toluene and chloroform and insoluble in pentane and water.

\section{Experimental part}

\subsection{Materials}

1. Commercial bulk polymerized PVC was obtained from ENIP (Skikda Algeria, type 4000M Kwert =65-66), its average molecular weights determined by Gel Permeation Chromatography were $M_{w}=149000 \mathrm{~g} / \mathrm{mole}$ and $M_{n}=71700 \mathrm{~g} / \mathrm{mole}$. It was used as the base polymer.

2. Tetrahydrofuran, ethanol and pentane were supplied from Prolabo (Paris, France) and used as good solvent and nonsolvents respectively.

3. Ethanol (Prolabo, France), ethylene glycol (Merck) and phenol (Fluka) were used as reagents.

*Corresponding author, e-mail: Hafida_mekki@yahoo.com

(C) BME-PT and GTE 
4. The preparation of Maghnite- $\mathrm{K}$ was carried out with the method described by Belbachir and coworkers [8-11], using $100 \mathrm{~g}$ of the raw Algerian clay (Maghnia; West Algeria) and $20 \mathrm{~g}$ of potassium hydroxide (KOH, Fisher Scientific). It was crushed for $20 \mathrm{~min}$ with a Prolabo ceramic balls grinder, then dried through baking at $105^{\circ} \mathrm{C}$ for $2 \mathrm{~h}$ and cooled to room temperature.

\subsection{Modifications of PVC}

PVC ( $3 \mathrm{~g} ; 48 \mathrm{mmole}$ ) was dissolved in $30 \mathrm{ml}$ of THF (solution 1).

48 mmole from each alcohol (ethanol $2.2 \mathrm{~g}$, ethylene glycol $3 \mathrm{~g}$ and phenol $4.5 \mathrm{~g}$ ) was mixed each one with $3 \mathrm{~g}$ of Maghnite- $\mathrm{K}$ and agitated for 15 minutes at room temperature and added to the solution 1.

After 4 hours the reactions were stopped, filtered and precipitated in cold ethanol. The modified polymers were purified using THF/Pentane (for sample with ethanol: copolymer 1) and THF/ Ethanol (for sample with ethylene glycol: copolymer 2, and phenol: copolymer 3).

The yield of the grafted PVC was optimized after 4 hours, and it was been between 50 and $83 \%$.

\subsection{Test methods}

${ }^{1} \mathrm{H}$ NMR spectra were recorded at room temperature, on a $300 \mathrm{MHz}$ Bruker Avance spectrometer, using deuterated DMSO or chloroform. as solvents. FT-IR measurements were performed on thin cast films of polymers using ATI MATTSON FT-IR spectrometer.

GPC measurements of the grafted PVC samples were carried out using a WISP 712, Waters Associates chromatograph, THF was used as solvent and the instrument was calibrated to a first approximation with polystyrene of known molecular weights. The flow rate of tetrahydrofuran was $10 \mathrm{ml} / \mathrm{min}$. Intrinsic viscosity measurements were performed on SEMATECH Viscologic TI 1 apparatus at $25^{\circ} \mathrm{C}$ using THF as solvent.

\section{Results and discussion}

Reaction of PVC with various alcohols in a THF solution gives primarily the corresponding copolymers in high transformation yield (Figure 1). The

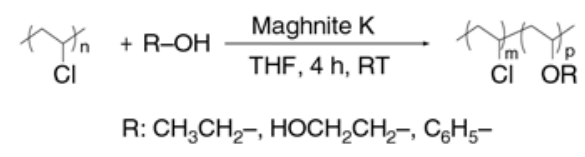

Figure 1. Grafting of PVC with various alcohols using Maghnite-K

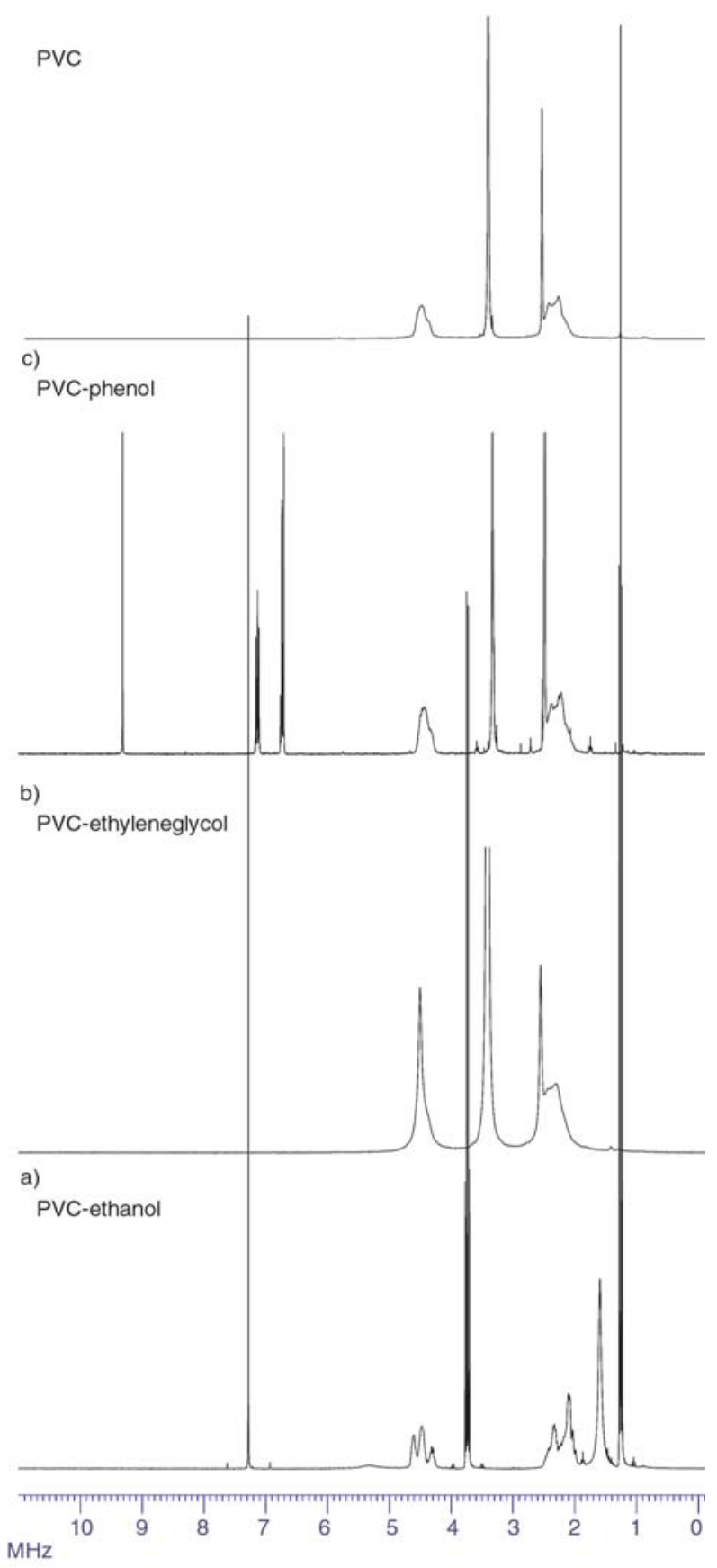

Figure 2. ${ }^{1} \mathrm{H}$ NMR spectra of alcohols grafting on PVC. a) PVC-g-ethanol $\left(\mathrm{CDCl}_{3}\right)$, b) PVC-g-ethylene glycol $\left(\mathrm{CDCl}_{3}\right)$ and c) PVC-g-phenol (DMSOd6)

resulting products are soluble in many organic solvents such as ethyl acetate, DMF, DMSO and THF. PVC itself is not soluble in ethyl acetate. 


\subsection{Characterizations of modified PVC}

The modification reactions were performed as indicated in experimental part section.

Samples were withdrawn, purified and analysed by ${ }^{1} \mathrm{H}$ NMR spectroscopy.

In Figure 2 the corresponding spectra of the modified PVCs are shown. With the appearance of alcohols proton peaks $[7,12]$.

1. In copolymer 1 (Figure 2a) two signals at $1.24 \mathrm{ppm}$ and $3.73 \mathrm{ppm}$ corresponding to $\mathrm{CH}_{3}$ and $\mathrm{CH}_{2}$ of ethanol respectively arise.

2. In copolymer 2 (Figure $2 \mathrm{~b}$ ) a signal at $3.74 \mathrm{ppm}$ appear corresponding to $\mathrm{CH}_{2}$ of ethylene glycol.

3. In copolymer 3 (Figure 2c) aromatic's signal (6.7-7.2 ppm) are appeared.

The variation of the chemical structure through the grafting of various alcohols onto the PVC was confirmed by FT-IR as shown in Figure 3.

The most significant change in each spectra of the grafted PVC was the appearance of the red-shifted (1042-1047 $\mathrm{cm}^{-1}$ ) absorption band due to the $\mathrm{C}-\mathrm{O}$ stretching vibration of ethers.

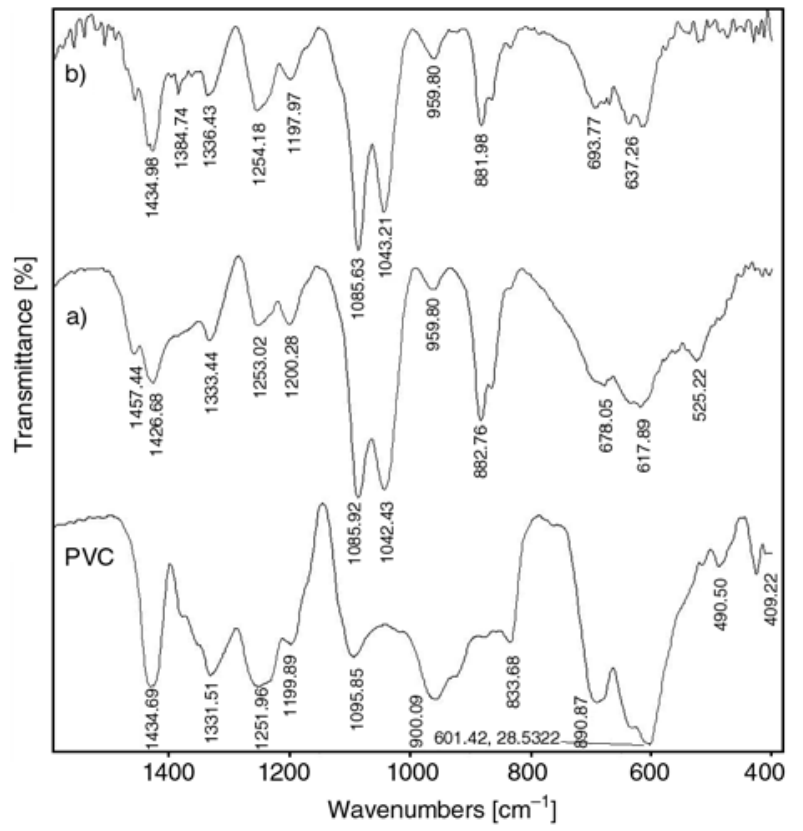

Figure 3. FT-IR spectra of alcohols grafting on PVC. a) PVC-g-ethanol and b) PVC-g-ethylene glycol
Also, the major characteristic peaks for PVC appeared at $720,760,800 \mathrm{~cm}^{-1}$ representing $\mathrm{C}-\mathrm{Cl}$ stretching bands.

It is important to note that, GPC analysis of the PVC and various copolymers shows a sharp decrease in the molecular weight in relation to that of the starting PVC in sample with phenol, and outstanding increase in sample with ethanol, a light increase in sample with ethylene glycol, as shown in Table 1.

Also comparison of the intrinsic viscosities (Table 1) of PVC and copolymers 1, 2 and 3 shows a same variations during modification with ethanol, ethylene glycol and phenol. At the moment it is not possible to suggest a mechanism for this process which appears to take place under very mild conditions. Further work is necessary to shed light on this.

\section{Conclusions}

This study reveals that:

PVC can be chemically modified by various alcohols using Maghnite- $\mathrm{K}^{+}$as catalyst without appreciable dehydrochlorination.

The PVC modified with alcohols is not explosive under ambient conditions.

The conversion rate of PVC to copolymer (VCEther) product is high $(\sim 63 \%)$.

It has been shown that ethanol; ethylene glycol and phenol are appropriate agents to introduce ether groups onto the PVC.

The presence of the ether groups in PVC changes markedly the chemical and physical properties of the systems.

\section{Acknowledgements}

We are grateful to Professor Samuel Lesko (Veeco Metrology Group, Dourdan, France) for the AFM analysis, Professor Alain Rameau (institut Charles Sadron, Strasbourg, France) for the GPC analysis and Malika Akeb, (LCP, université d'Oran, Algérie) for the NMR analysis of copolymers.

Table 1. $M_{n}, M_{w}, I$, intrinsic viscosity and color of copolymer 1, 2 and 3

\begin{tabular}{|l|c|c|c|c|c|}
\hline \multicolumn{1}{|c|}{ Samples } & $\mathbf{M}_{\mathbf{n}}{ }^{\mathbf{a}}$ & $\mathbf{M}_{\mathbf{w}}{ }^{\mathbf{}}$ & $\mathbf{I}^{\mathbf{b}}$ & Intrinsic viscosity $^{\mathbf{c}}[\mathbf{m l} / \mathbf{g}]$ & Color \\
\hline PVC & 71700 & 149100 & 2.00 & 8.12 & white \\
\hline Copolymer 1 & 76400 & 159600 & 2.09 & 8.65 & white \\
\hline Copolymer 2 & 89500 & 172300 & 1.90 & 14.74 & white \\
\hline Copolymer 3 & 25800 & 43200 & 1.57 & nd & yellow \\
\hline
\end{tabular}

nd: not determined

adetermined by GPC with polystyrene standard; b $I$ - polydispersity index $\left(M_{w} / M_{n}\right)$; cin THF at $25^{\circ} \mathrm{C}$ 


\section{References}

[1] Reinecke H., Mijangos C.: Synthesis and characterization of poly(vinyl chloride)-containing amino groups. Polymer, 38, 2291-2294 (1997).

[2] Kim D S., Kang J S., Kim Y. K., Lee Y. M.: Surface modification of a poly (vinyl chloride) membrane by UV irradiation for reduction in sludge adsorption. Desalination, 146, 301-305 (2002).

[3] Anzai. J. I., Liu. C. C.: Potentiometric response of $\mathrm{PVC} /$ crown ether membrane electrodes to nonionic organic compounds. Sensors and Actuators B: Chemical, 5, 171-172 (1991).

[4] Bromberg L., Levin G., Kedem O.: Transport of metals through gelled supported liquid membranes containing carrier. Journal of Membrane Science, 71, 4150 (1992).

[5] Bicak N., Sherrington N. C., Bulbul H.: Vinylamine polymer via chemical modification of PVC. European Polymer Journal, 37, 801-805 (2001).

[6] Herrero M., Tiemblo P., Reyes-Labarta J., Mijangos C., Reinecke H.: PVC modification with new functional groups. Influence of hydrogen bonds on reactivity, stiffness and specific volume. Polymer, 43, 26312636 (2002).
[7] Tiemblo P., Guzman J., Riande E., Mijangos C., Reinecke H.: Effect of physical aging on the gas transport properties of PVC and PVC modified with pyridine groups. Polymer, 42, 4817-4824 (2002).

[8] Belbachir M., Bensaoula A.: Composition and method for catalysis using bentonites. U S Patent 7094823 , USA (2006).

[9] Harrane A., Meghabar R., Belbachir M.: Polymerization of $\varepsilon$-caprolactone using a montmorillonite clay as catalyst. Designed Monomers and Polymers, 8, 11-24 (2005).

[10] Yahiaoui A., Belbachir M., Soutif J. C., Fontaine L.: Synthesis and structural analyses of poly (1, 2-cyclohexene oxide) over solid acid catalyst. Materials Letters, 59, 759-767 (2005).

[11] Reguieg F., Sahli N., Belbachir M., Lutz P. J.: Onestep synthesis of bis-macromonomers of poly(1,3dioxolane) catalysed by maghnite- $\mathrm{H}^{+}$. Journal of Applied Polymer Science, 99, 3147-3152 (2006).

[12] Kennedy J. P., Zhengjie P.: Addition of unsaturated hydrocarbons to poly(vinyl chloride) and functionalization thereof. U S Patent 6831133, USA (2004). 